\title{
NUEVAS CONCEPCIONES ESPACIALES EN THE TURN OF THE SCREW
}

\author{
José Manuel Correoso Rodenas \\ Universidad de Castilla-La Mancha \\ JoseManuel.Correoso@uclm.es
}

\section{RESUMEN}

El objetivo del presente artículo es poner de manifiesto los juegos espaciales creados por el escritor neoyorkino Henry James en su novela breve The Turn of the Screw. Más allá de ser considerada como una simple novela de fantasmas, se observa como la composición espacial de la obra participa de una serie de características muy marcadas. Asimismo, algunos elementos presentes en la misma contribuyen a acentuar el carácter innovador y vanguardista en lo que a los espacios se refiere, impregnando toda la historia.

Palabras Clave: Henry James, The Turn of the Screw, espacios, composición matemática, composición vertical.

\section{ABSTRACT}

The purpose of this essay is to highlight the spatial games created by Henry James in his novella The Turn of the Screw. Considered beyond a simple ghost story, it can be appreciated how the whole composition of spaces is marked with extraordinary and unique characteristics. In addition, some of the elements present in the story contribute to highlight the innovative and forefront attractiveness of this literary work.

KEY WORDS: Henry James; The Turn of the Screw; spaces; mathematical composition; vertical composition. 


\section{INTRODUCCIÓN}

La literatura gótica norteamericana hunde sus raíces en los años finales del siglo XVIII ${ }^{1}$, experimentando un gran desarrollo durante el XIX. Uno de los primeros ejemplos se encuentra en la obra de Charles Brockden Brown (1771-1810), con su novela Wieland; or, The Transformation (1798). Unos años después, otro estadounidense, Isaac Mitchell (1758-1812), aportaría un nuevo ejemplo de "American Gothic" con una obra que ha pasado prácticamente desapercibida para el público y para la crítica hasta nuestros días, y que se conoce gracias a una edición pirata que Daniel Jackson, Jr. publicó en 1811: Alonzo and Melissa (1804), posteriormente incluida en la novela The Asylum (1811). Si se toma como referencia el llamado "gótico de primera generación", estos serían los ejemplos más sobresalientes que Estados Unidos presenta, a excepción, quizá, de algunas de las obras de juventud de Washington Irving. El resto de los magníficos ejemplos del gótico norteamericano trasciende los límites cronológicos - 1820 - marcados para esta primera hornada de obras. Sin embargo, atendiendo simplemente a estas primeras piezas, podemos apreciar lo que, según algunos críticos, va a ser el principal rasgo del gótico estadounidense: el hecho de que las obras abandonen las ambientaciones míticas de castillos, abadías, bosques tenebrosos, etc., para situarse en casas burguesas de la ciudad o de la campiña americana, cuyos protagonistas van a ser personajes verosímiles alejados del efectismo europeo. En palabras de Fred Botting:

[...] the bourgeois family is the scene of ghostly return, where guilty secrets of past transgression and uncertain class origins are the sources of anxiety... Though the grand gloom of European Gothic was inappropriate, the commonplaces of American culture was full of Little mysteries and guilty secrets from communal and family pasts (en Wheatley 2006:123).

Botting introduce aquí dos de las notas dominantes que van a poblar el gótico norteamericano: el núcleo familiar como foco de los miedos y de un pasado perturbador que retorna una y otra vez, y la herencia religiosa de corte puritano. En efecto, si se atiende a la cantidad de obras literarias que siguen los postulados del gótico, es posible apreciar que los fenómenos paranormales característicos del género, si son padecidos por una familia, es debido a un pasado impío o a la comisión de algún gran pecado que es necesario confesar y purgar $^{3}$. Sin embargo, Botting no ha sido el primer crítico en apuntar esta eventualidad. Ya en el propio siglo XIX, autores de gran renombre, como pueden ser el propio Henry James, se hicieron eco de esta característica definitoria del gótico allende el Atlántico. Helen Wheatley dice al respecto:

Henry James proclaimed in 1865 that American Gothic literature "was connected at a hundred points with the common objects of life"(Davenport-Hines, 1998:267), and contemporary critics have also recently taken up James' stance. Fred Botting, for example, characterizes the early American Gothic as a "domestication" of the European Gothic [...]. Botting details the fact that the canonical texts of the American Gothic, such as Charles Brockden Brown's Wieland (1798),

\footnotetext{
${ }^{1}$ Véase, por ejemplo, Richard Ruland (1992): From Puritanism to Postmodernism. A History of American Literatue. Nueva York: Penguin.

2 Aquel desarrollado entre finales del siglo XVIII y 1820 aproximadamente.

3 Aunque también, y posteriormente, cinematográficas. Véase An American Haunting, dirigida por Courtney Solomon (2006).
} 
Nathaniel Hawthorne's The House of the Seven Gables (1851) and Edgar Allan Poe's "The Fall of the House of Usher" (1834), all focus on familiar psychodramas, with each of these keys texts taking the ancestral home as their setting in narratives that focus the resurfacing of family guilt (2006:123).

Como se aprecia en el fragmento anterior, durante las décadas centrales del siglo XIX, escritores tan destacados como Washington Irving (1783-1859), Edgar Allan Poe (1809-1849), Nathaniel Hawthorne (1804-1864) o Herman Melville (1819-1891) produjeron ejemplos de literatura gótica que hoy en día están considerados entre las obras cumbres de la literatura universal, a pesar de que no todos ellos eran autores eminentemente góticos, pues, como es bien sabido, durante este periodo el gótico se va diluyendo y confundiendo con el resto de géneros literarios surgidos a lo largo de la centuria.

Se llega, así, a las últimas décadas del siglo, en las que, tanto en Europa como en los Estados Unidos, la literatura fantástica o de corte sobrenatural vuelve a estar de moda. El cambio de siglo es, una vez más, un momento propicio para el desarrollo de los miedos y los anhelos más ocultos de la población. En este momento, a caballo entre las letras británicas y americanas, surge la figura de Henry James (1843-1916). La mayor parte de su producción narrativa, dejando de lado sus escritos de crítica literaria o sus escarceos en el mundo del teatro, participa de lo que se ha dado en llamar el "realismo psicológico". El autor no está interesado en los acontecimientos históricos o sociales que rodean la vida de los personajes, sino en su mundo interior, en todo el devenir de ideas, pensamientos, sentimientos y aflicciones que cruzan sus mentes y que, de algún modo, configuran sus trayectorias; mundo interior de los personajes que acaba por condicionar sus acciones y, en definitiva, su existencia ${ }^{4}$. En cuanto a su temática, las relaciones bilaterales Estados Unidos-Europa aparecen como nota dominante, al menos en sus dos primeras etapas literarias (hasta 1900 aproximadamente). James hace de esta dualidad un reflejo de su propia vida y, además, de todo un compendio mitológico-alegórico donde enfrenta la experiencia (en sentido negativo) de los europeos con la ingenuidad americana. Los personajes americanos (Isabel Archer, protagonista de The Portrait of a Lady, sería prototípica) sucumben ante la corrupción de la sociedad europea. Pero Henry James también produjo literatura de corte gótico; algunos de sus textos han pasado a la posteridad como cuentos o narraciones breves ("Sir Edmund Orme" — 1891—, "Owen Wingrave" — 1892 — o "The Friends of the Friends" — 1896-), mientras que otros (What Maisie Knew -1897_ y The Turn of the Screw - 1898 - ) fueron desarrollados en forma de novelas cortas. Todos ellos presentan el denominador común de participar plenamente de las teorías expuestas más arriba: la aparición de un fantasma que "vuelve" y se manifiesta a los presentes para darles a conocer algún aspecto relativamente desconocido (u oculto) sobre el pasado. Este elemento no es novedoso de la narrativa de Henry James, sino que ya se había manifestado en obras anteriores pertenecientes a la tradición inglesa. Durante el período victoriano, autores como Elizabeth Gaskell (1810-1865) o el propio Charles Dickens (1812-1870) recurrieron a la inclusión de estos "fantasmas", reales o no, para organizar la composición de varias de sus obras, entre las que se destaca “The Old Nurse’s Story”, de Elizabeth Gaskell (1852).

${ }^{4}$ Se le considera, por tanto, un precursor del monólogo interior que, ya en el siglo xx, desarrollará James Joyce, entre otros autores significativos, como Virginia Woolf. 


\section{APROXIMACIÓN A THE TURN OF THE SCREW}

Esta es una de las obras más conocidas y populares de la producción jamesiana, tanto por la agilidad de la narración como por su carácter perturbador y las implicaciones presentes en sus páginas. La obra fue publicada, de forma periódica, en la Collier's Magazine ${ }^{5}$, entre enero y abril de 1898, y parece tener su origen en una conversación que James mantuvo en 1895 con su buen amigo Edgard White Bennett, arzobispo de Canterbury, tal y como se narra en sus Notebooks (273.1) ${ }^{6}$. Sea esto cierto o no, lo realmente importante es que, con esta novela breve, Henry James alcanzó un nivel de perfección que, según algunos críticos, no se vería superado por ninguna otra obra del autor (Bravo Castillo: 2010:1084). Ya en aquel momento surgieron voces aclamando The Turn of the Screw como una magistral muestra de la literatura sobrenatural. Tomemos el ejemplo del testimonio de Edith Wharton: "For imaginative handling of the supernatural no one, to my mind, has touched Henry James in 'The Turn of the Screw" (1997:3). Si se atiende a la biografía del propio James, el hecho de escribir un relato de fantasmas como The Turn of the Screw en 1898 responde a algunos factores que deben ser tenidos en cuenta. Por ejemplo, según apunta Santiago Rodríguez Guerrero-Strachan: "También en la familia James se dieron casos de neurastenia, alcoholismo, suicidio o comportamientos derrotistas" (2011:17). El mismo crítico señala que la fallida experiencia teatral de James le llevó a buscar nuevas formas de expresión; The Turn of the Screw supone, por tanto, un avance de los derroteros que va a seguir la obra posterior del autor pues - según su opinión- en Otra vuelta de tuerca asoma ya su posterior obsesión por la muerte y la enfermedad (Rodríguez Guerrero-Strachan 2011:22).

En cuanto a su clasificación, en lo que a la estructura se refiere, y debido a su extensión (unas 100 páginas \pm ), se torna difícil catalogar esta obra, pues se acerca a tres formatos: la novela, la novela corta y el relato largo. Las ediciones contemporáneas vacilan hacia un lado o hacia otro dependiendo de los intereses editoriales y del resto de obras que aparezcan en el volumen, pues raramente se presenta sola. Si aparece con algunos de los cuentos del autor, tiende a considerársela como tal, siendo calificada de novela o novela corta si aparece en compañía de otras obras, como Daisy Miller ${ }^{7}$. Ya en 1898, esta cuestión suscitó una polémica entre críticos y editores; polémica que se vio azuzada por las palabras del propio James:

I need scarcely add after this that it is a piece of ingenuity pure and simple, of cold artistic calculation, an amusette to catch those not easily caught (the 'fun' of the capture of the merely witless being ever but small, the jaded, the disillusioned, the fastidious ("Preface" a The Turn of the Screw. 2008: 1) ${ }^{8}$.

\footnotetext{
${ }^{5}$ Revista norteamericana editada por Peter Fenelon Collier, y fundada en 1888.

${ }^{6}$ En dicha conversación Bennett le contó a James una historia acaecida veinte años antes a una conocida suya. Véase, a este respecto, Juan Bravo Castillo: Grandes hitos de las historia de la novela euroamericana. Vol. II. El siglo XIX: los grandes maestros. Madrid: Cátedra, 2010, pp. 1084-1088.

7 Téngase en cuenta que Daisy Miller también se ha considerado como relato en algunas ediciones, como la de Austral de 2011.

${ }^{8}$ Nótese, en algunos aspectos, como la referencia a la composición calculada, las similitudes con The Philosophy of Composition (1846), de Edgar Allan Poe.
} 
Si se atiende tanto a la complejidad estructural, al desarrollo interno de los personajes, o a la coordenada espacio-temporal, se puede afirmar que The Turn of the Screw vendría a situarse entre las novelas breves (o novellas) de Henry James. La trama adquiere una complejidad mayor que en los mencionados cuentos de fantasmas. Por lo tanto, es factible afirmar que se trata de un ejemplo literario distinto de aquellos ${ }^{9}$.

\section{ANÁLISIS DE LA COORDENADA ESPACIAL}

En lo que a la configuración espacial de la obra se refiere, es de destacar, como primera característica, que Henry James sigue un modelo cuasi matemático, jugando con una serie de analogías. Ninguno de los detalles de la obra ha sido dejado al azar, sino que está cuidadosamente calculado para ofrecer esa visión total de conjunto que los críticos admiran a la hora de calificar este amusette entre las obras más sobresalientes del autor. The Turn of the Screw se divide en un prólogo y veinticuatro capítulos. La obra se abre, por tanto, con un prólogo en el que aparece un grupo de conocidos en una típica reunión burguesa ${ }^{10}$. Durante esta soirée los presentes deciden entretenerse contando historias de fantasmas. De hecho, en el momento en el que el lector se asoma a la obra, acaban de finalizar una ${ }^{11}$. La escena se tiñe de tintes hogareños, pues la acción tiene lugar en una casa — eso sí, una mansión— particular, del campo o de la ciudad, y no en un fastuoso palacio de corte medieval ni en los lúgubres salones de una abadía abandonada. Conforme avanza la conversación, los personajes reunidos, entre ellos el primer narrador, se animan a escuchar una historia que uno de ellos, el segundo narrador, va a relatar. Esta historia le fue referida por una institutriz conocida suya, y es mostrada en forma de confesión, en primera persona (tercer narrador).

Esta "segunda" parte de la historia comienza con una joven institutriz que obtiene un empleo en una casa de campo con la condición de que no debe molestar al dueño. Una vez allí se percata de que la vida de los niños a su cargo está rodeada de misterios. En un momento dado, la joven percibe la presencia de un hombre y, posteriormente también de una mujer, a los que no conoce. Gracias a las explicaciones del ama de llaves, llega a la conclusión de que se trata de Peter Quint y Miss Jessel, recientemente fallecidos. Estos dos personajes, al parecer, ejercieron en vida una perniciosa influencia sobre Miles y Flora (los niños). La institutriz asume que han vuelto para completar su malévola obra y se arroga el papel de salvadora de las criaturas. Al final de la obra, el joven Miles muere en sus brazos después de tener, supuestamente, un encuentro con el difunto Peter Quint. Debe remarcarse que durante el transcurso de los hechos es sólo la institutriz quien establece contacto con los supuestos fantasmas, lo que contribuye a acentuar el carácter ambiguo de toda la composición.

9 Debido a la dificultad que presenta la dicotomía novela/cuento, se remite al lector a algunos de los ejemplos de la bibliografía, especialmente las obras de Mariano Baquero Goyanes.

${ }^{10}$ Véase el paralelismo con otras obras producidas en la misma época, como el comienzo de Heart of Darkness (1899) de Joseph Conrad; o el relato "La mujer alta" del español Pedro Antonio de Alarcón o la mencionada "The Old Nurse's Story", de Elizabeth Gaskell.

${ }^{11}$ Esta obra que acaban de referirse no es otra que la propia novela de James What Maisie Knew. 
El hecho de que el personaje principal sea una institutriz es bastante significativo por sí mismo. Las institutrices habían gozado, durante todo el siglo XIX, de un gran predicamento en la literatura ${ }^{12}$. En cuanto a la espacialización (coordenada espacial y tratamiento de los espacios) de la novela, la introducción de una institutriz es especialmente interesante. Por medio de este personaje prototípico, Henry James compone una estructura vertical que, como veremos, va a ser una nota dominante a lo largo de la obra. Como dice Lusting: "An outsider within the family, the nineteenth-century governess belonged neither above nor below stairs, neither exclusively with the children nor with the adults, neither amongst women nor with men" (2008:XXIII). La sociedad victoriana, de la que la casa es un reflejo a pequeña escala (un microcosmos, se podría decir) se hallaba estructurada jerárquicamente. No obstante, es posible identificar elementos conectores entre los distintos estamentos que la componían. Una institutriz representaba uno de estos conectores. Como se verá más adelante, este es el primer "rompimiento"13 espacial que Henry James construye a lo largo de la novella.

La historia que la institutriz confiesa por boca del segundo narrador es la del período que pasó trabajando en la finca rústica de Bly. No obstante, antes de llegar a la mansión a finales del capítulo I, la (entonces) aspirante a institutriz realiza una visita al que va a ser su jefe. Allí se le explican las condiciones de su contrato:

That she should never trouble him — but never, never: neither appeal nor complain nor write about anything; only meet all questions herself, receive all moneys from his solicitor, take the whole thing over and let him alone (2008:122).

Se observa, en este fragmento, otra característica que va a condicionar el desarrollo de la obra y que se halla relacionada con la coordenada espacial: el aislamiento. En efecto, ninguna noticia de lo ocurrido en Bly debe trascender sus límites; los personajes se van a encontrar, en todo momento, solos ante el peligro que representa lo sobrenatural. Como apunta Rodríguez Guerrero-Strachan: "Y una vez más la casa como lugar acotado en el que los personajes viven fuera del tiempo" (2011:30). Antes de que ningún personaje entre en contacto con la mansión, los hilos compositivos principales, en lo que al tratamiento del espacio se refiere, aparecen expuestos: rompimiento de espacios, construcción en vertical y aislamiento total.

Al final del capítulo I, el lector "contempla", a través de los ojos de la institutriz, la mansión:

[...] it was a big ugly antique but convenient house, embodying a few features of a building still older, half-displaced and half utilised, in which I had the fancy of our being almost as lost as a handful of passengers in a great drifting ship. Well, I was strangely at the helm! (2008:127).

Esta primera descripción de la mansión contrasta con los perturbadores acontecimientos que van a tener lugar en las páginas que siguen. Sin embargo, James juega con esa tonalidad de frontera de la que toda la obra anda revestida: "half-displaced and half utilised". La comparación que se establece con un barco ("a great drifting ship"), es decir, con un espacio en

12 Jane Eyre, de Charlotte Brontë, queda como ejemplo paradigmático.

${ }_{13}$ Se ha decidido adoptar este término, propio de vocabulario artístico, por los posibles paralelismos que puede tener con la obra, que serán expuestos más adelante. 
movimiento, también viene a unirse la ruptura a la que se ha aludido anteriormente. Pero la mansión pronto se verá acompañada de un aura gótica, comparable a algunos de los ejemplos clásicos del género, como la propia institutriz apunta: "Was there a secret at Bly —a mystery of Udopho ${ }^{14}$ or an insane, an unmentionable relative kept in unsuspected confinment? ${ }^{15}$ " (2008:138).

La vivienda, por su parte, no se halla desprovista de "habitantes", sino que en ella viven Mrs. Grose (el ama de llaves), Luke (un criado) y toda una suerte de criados, doncellas, etc., anónimos. A todos ellos habría que añadir a dos niños, Miles y Flora que, al igual que la institutriz, representan un tipo de personaje ajeno al ambiente circundante, pues son los señores pero, a su vez, niños a las órdenes del servicio que su tío ha puesto en la casa. En un principio, el contacto entre las dos mujeres que gobiernan la casa es frío y distante. Mrs. Grose, por ejemplo, trata de rehuir algunas de las preguntas de la recién llegada. Sin embargo, al pie de una escalera, consiguen entablar una fructífera conversación: “[...] we went down together and at the bottom I detained her" (2008:130). La escalera que cruza la casa posee un elevado simbolismo y una alta significación en la obra. En cuanto símbolo vendría a actuar como la propia institutriz, de nexo de unión entre espacios diferentes; pero como elemento estructural se aprecia que es en ella en la que tienen lugar algunos de los acontecimientos trascendentales que rodean la trama. Habría que atender al simbolismo que la escalera ha presentado ya desde la Antigüedad, por ejemplo, en el sueño de Jacob:

Llegó [Jacob] a un determinado lugar y se quedó allí a pernoctar, porque ya se había puesto el sol. Tomando una piedra de allí mismo, se la colocó por cabezal y se echó a dormir en aquel lugar. Y tuvo un sueño: una escalinatas, apoyada en la tierra, con la cima tocaba el cielo. Ángeles de Dios subían y bajaban por ella (Génesis 28:11-12. 2011:35).

De interés es, asimismo, su utilización por parte de la Masonería como símbolo de unión entre lo terrenal y lo celeste ${ }^{16}$, pero también como vía para alcanzar la perfección. La escalera de The Turn of the Screw sirve de nexo de unión entre el mundo real y el sobrenatural pues, en dos ocasiones, la institutriz "ve" a alguno de los "fantasmas" en este espacio de la casa, primero a Peter Quint:

I came within sight of the tall window that presided the great turn of the staircase. [...]. My candle, under a bold flourish, went out, and I perceived, by my uncovered window, that the yielding dusk of earliest morning rendered it unnecessary. Without it, the next instant, I knew that there was a figure on the stair (2008:170);

y, en el siguiente capítulo, a Miss Jessel:

I just missed, on the staircase, nevertheless, a different adventure. Looking down it from the top I once recognized the presence of a woman seated on one of the lower steps with the back presented to me, her body half-bowed and her head, in an attitude of woe, in her hands (2008:173).

\footnotetext{
${ }^{14}$ En referencia a la obra de Ann Radcliffe.

15 En referencia a Bertha Mason, personaje de Jane Eyre.

${ }^{16}$ Muy en relación con la simbología que se otorga a la Virgen María en la Iglesia Católica.
} 
La finca se halla rodeada de la típica campiña inglesa, por la que la nueva institutriz gusta de pasear. En una de estas caminatas, el orden y la tranquilidad de Bly serán destruidos. Henry James pinta una magnífica estampa del lugar en el que aparece Peter Quint por primera vez:

The tower was one of a pair — square incongruous crenelled structures - that were distinguished, for some reason, though I could see Little difference, as the new and the old. They flanked opposite ends of the house and were probably architectural absurdities, redeemed in a measure indeed by not being wholly disengaged nor a height too pretentious, dating, in their gingerbread antiquity, from a romantic revival that was already a respectable past (2008:135-136).

La casa se halla flanqueada por dos torres casi idénticas, acentuando la sensación de aislamiento, pero también la composición matemática de la obra: “[...] there are two towers, two children, two scenes by the lake, two by the dining-room window, and so on" (Lusting, 2008:XXI). Por otro lado, esas torres vienen a servir, de igual modo que la institutriz y la escalera, de elemento conector. Sólo que, en este caso, las realidades conectadas serán más distantes. No en vano, será este el lugar en el Peter Quint se manifieste por primera vez: en lo alto de una de las torres, remarcando esa estructura vertical apuntada más arriba. Una torre también sirve como elemento disuasorio o, lo que es más importante, de vigilancia ${ }^{17}$. Por lo tanto, cabría preguntarse: ¿quién es el vigilante y quién el vigilado? Por otro lado, las torres que hay en Bly no son torres cualquiera, sino que una de ellas es nueva y la otra vieja ("from a romantic revival that was already a respectable past"). El simbolismo de esta clasificación temporal es patente: la torre antigua sería la materialización de Peter Quint y Miss Jessel, personajes del pasado que darían paso a la torre nueva, la de la nueva institutriz. La casa, enmarcada entre ambas, será el escenario en el que presente y pasado libren la batalla definitiva por el control de las almas (aunque también podría decirse de los cuerpos) de Miles y Flora. Henry James juega, de forma magistral, no sólo con los espacios físicos, sino también con todo aquello que es "espacializable". El tratamiento que muchas veces se hace del tiempo es similar en sus descripciones al de los espacios, creando un momento/lugar fronterizo ${ }^{18}$. Por ejemplo, mediante la utilización del crepúsculo: “[...] in the clear twilight, [...] The gold was still in the sky, the clearness in the air, and the man who looked at me over the battlements was as definite as a picture in a frame" (2008:136). Como se aprecia en este fragmento, las torres se han transformado en "battlements", dotándolas ya del significado belicoso que van a tener. Por otro lado, Mr. Quint, dice la institutriz, parece como un retrato, inevitablemente relacionado con un espejo ${ }^{19}$. Entonces, los "fantasmas" no serían sino el reflejo del alma (pasiones y anhelos más íntimos) de la institutriz, como ha señalado buena parte de la crítica (Bravo Castillo 2010:1087). Otros ejemplos de esta utilización de los matices de color para crear una sensación de espacio, que variará dependiendo del estado de ánimo de los personajes o de la sensación que se quiera transmitir, se vuelve a ver más adelante; por ejemplo, cuando Miles llega del internado y la nueva habitante de Bly intenta esclarecer los motivos de

${ }^{17}$ Recuérdese, por ejemplo, el capítulo inicial de La Regenta de Leopoldo Alas "Clarín”, en que Fermín de Pas vigila a Ana Ozores desde lo alto del campanario de la catedral de Vetusta.

${ }_{18}$ Como ya hiciera Joseph Sheridan Le Fanu en la ambientación de su inmortal Carmilla.

${ }_{19}$ Resulta inevitable establecer relación con otros retratos relacionados con lo sobrenatural, como el poeniano "The Oval Portrait" o el wildeano The Picture of Dorian Gray. 
su expulsión: "deep obscurity continued to cover the region of the boy's conduct at school" (2008:140).

Si se avanza en la trama, se van descubriendo nuevas estancias o lugares de la mansión aunque, debido a que la narradora es la institutriz, después del primer encuentro "sobrenatural" las descripciones serán más tétricas o pesimistas. Rara vez un cielo despejado o un "clear twilight" volverá a aparecer sobre Bly. Todo serán nubes de sospecha, oscuridades que presagian monstruos y la presencia constante de aquello que no existe, o que es demasiado real como para ser admitido ${ }^{20}$. Cuando Mrs. Grose y la joven hablan acerca del hombre de la torre y esta le refiere cuándo tuvo lugar el encuentro, el ama de llaves espetará: "Almost at dark" (2008:145), en contraposición con el cielo dorado descrito páginas antes. Todo esto se completa con el paso de las estaciones. Pronto, el verano dará paso al oscuro otoño inglés:

The summer had turned, the summer had gone; the autumn had dropped upon Bly and had blown out half our lights. The place, with its grey sky and withered garlands, its bared spaces and scattered dead leaves, was like a theatre after the performance —all strewn with crumpled playbills (2008:185).

El buen tiempo y la calma atmosférica sólo habrán de regresar cuando la acción se consume y el niño Miles fallezca en brazos de su cuidadora. En efecto, la última frase del libro reza: "We were alone with the quiet day, and his Little heart, dispossessed, had stopped" (2008:236).

No sólo la casa es escenario de los eventos de carácter "sobrenatural" que tienen lugar, pues también los alrededores van a ser escenarios para estos hechos, como puede ser, por ejemplo, el "encuentro" de Flora y Miss Jessel junto al lago. Un lago cuya descripción es la de oscuridad, a pesar de ser media tarde: "The old trees, the thick shruberry, made a great and pleasant shade" (2008: 154); un lago que se compara con el mar por lo que representa ${ }^{21}$ y por lo que (supuestamente) hay al otro lado de sus aguas ${ }^{22}$ : "Suddenly, amid these elements, I became aware that on the other side of the Sea of Azof we had an interested spectator" (2008:154). Pero no es un mar cualquiera, es el Mar de Azov, un mar en los confines de Europa, separando la civilización de la barbarie. Llegados a este punto, todos los actores se encuentran sobre el escenario. Ahora sólo hay que hacerlos bailar, y Henry James construye la pista de baile de forma difícilmente igualable en todos los sentidos. No sólo la posición física es importante, como ya se ha comentado, sino la posición que uno guarda dentro de la férrea jerarquía victoriana. Una vez más, la construcción en vertical sirve para ilustrar la naturaleza de las relaciones entre Peter Quint y Miss Jessel:

““[...] there was something between them.'

'There was everything.'

'In spite of the difference-?'

'Oh of their rank, their condition' -she brought it woefully out. 'She was a lady.'

\footnotetext{
${ }^{20}$ Recuérdese el grabado de Goya.

${ }^{21} \mathrm{El}$ mar ha sido uno de los símbolos tradicionales de la muerte en la literatura universal de todos los tiempos. Por citar un ejemplo español, recuérdense las Coplas a la muerte de su padre, de Jorge Manrique.

${ }^{22}$ En relación al elemento de la escalera, no debe olvidarse, por ejemplo, el mito de Caronte y la Laguna Estigia.
} 
I turned it over; I again saw. 'Yes-she was a lady.'

'And he so dreadfully below,' said Mrs Grose" (2008:159).

He ahí la composición de un nuevo espacio paralelo o complementario a los de la mansión: el espacio social, que también es explotado en la literatura jamesiana ${ }^{23}$.

Más arriba se apuntaba al paralelismo que la propia institutriz establece con Jane Eyre, al mencionar la posibilidad de que en Bly se encuentre un familiar confinado debido a una locura inconfesable. En el capítulo IX, de nuevo, la narradora vuelve a pagar ese pequeño homenaje a la obra de Charlotte Brontë, cuando nos ofrece tanto la descripción de la biblioteca de la mansión como la de su propio dormitorio:

There was a roomful of books at Bly — last century fiction some of it, [...]. I remember that the book I had in my hand was Fielding's 'Amelia'; also that I was wholly awake. I recall further both a general conviction that it was horribly late and a particular objection to looking at my watch. I figure finally that the white curtain draping, in the fashion of those days, the head of Flora's little bed, shrouded, as I had assured myself long before, the perfection of childish rest. I recollect in short that though I was deeply interested in my author I found myself, at the turn of a page and with his spell all scattered, looking straight up from him and hard at the door of my room. There was a moment during which I listened, reminded of the faint sense I had had, the first night, of there being something undefinably astir in the house, and noted the soft breath of the open casement just move the half-drawn blind (2008:169).

Leyendo estas líneas, resulta fácil recordar a la joven Jane en la mansión de Thornfield, especialmente en los momentos de reclusión en su cuarto. En el fragmento citado también se puede apreciar una característica de los espacios góticos: el hecho de que, a lo largo del siglo XIX, los espacios tiendan a interiorizarse y la sensación de opresión y constricción de los sentidos que antes producían castillos y abadías, ahora sea creada por la propia mente de los personajes (o los lectores). En esta escena, se observa cómo la institutriz empieza a sentir una "presencia" en el silencio y oscuridad de su alcoba, una sensación que, poco a poco, se apodera de ella y que llega a modificar la realidad. También se ha de tener en cuenta que el dormitorio de la institutriz es, en un momento dado, escenario de uno de los encuentros con lo sobrenatural: "Seated at my own table in the clear moonday light I saw a person whom, without my previous experience, I should have taken at the first brush for some housemaid who might have stayed at home to look after the place" (2008:195). Esa desconocida no es otra que Miss Jessel.

Hay en la obra una escena, sin embargo, que viene a ser el compendio de las principales características espaciales de The Turn of the Screw (composición vertical, matemática y unión de planos de la realidad). La escena en cuestión es aquella con la que concluye el capítulo X, en la que la institutriz se halla contemplando los jardines desde su ventana de su habitación ${ }^{24}$ $\mathrm{y}$ ve al joven Miles mirando hacia arriba:

The moon made the night extraordinarily penetrable and showed me on the lawn a person, diminished by distance, who stood there motionless and as if fascinated, looking up to where I

\footnotetext{
${ }^{23}$ Recuérdese la historia narrada en The American, por ejemplo.

${ }^{24}$ Que se haya en una de las torres anteriormente descritas.
} 
had appeared - looking, that is, not so much straight at me as at something that was apparently above me. There was clearly another person above me- there was a person on the tower; but the presence on the lawn was not the least what I had conceived and had confidently hurried to meet. The presence on the lawn —I felt sick as I made it out— was poor little Miles himself (2008:176).

El análisis de esta escena es especialmente interesante porque en ella confluyen algunos elementos que son muy importantes para entender la composición total de la obra. Por un lado, se aprecia que Henry James organiza una estructura en tres planos (Miles en el suelo, la institutriz en una ventana de la torre, alguien — supongamos que Quint - en lo alto de la misma). Esto podría interpretarse como una representación gráfica de esa situación de las institutrices en la Inglaterra victoriana expuesta más arriba, a medio camino entre los niños y los adultos. Otra posible interpretación es que la intención de James sea la de mostrar que la joven empleada es la encargada de unir a Miles y a Quint, como sucede en esta escena y como, supuestamente, acontecerá también en la que cierra el libro actuando, así, como psicopompo $^{25}$. Por otro lado, atendiendo a la situación de Miles, se puede observar que él es visto por los dos personajes (si existen) que están en la torre, uno del mundo de los vivos y otro del de los muertos. Por lo tanto, en esta escena, Miles estaría cumpliendo la función de escalera celeste (o, más bien, demoníaca), uniendo ambas realidades.

A lo largo de la novela aparecen otras tipologías espaciales que también merecen atención. Por ejemplo, hay una iglesia, único lugar fuera de Bly que los personajes visitan. Sin embargo, la parroquia que se muestra en The Turn of the Screw es descrita como un lugar ajeno a las vidas de los habitantes y a los sucesos que están teniendo lugar en el señorío. También se debe tener en cuenta que la iglesia, por su carácter exorcista, de intercesora entre el mundo terrenal y el divino y de protectora contra las "fuerzas del mal”, quizá fuera el único medio para librarse de las "apariciones", pero debe recordarse que la institutriz nunca llega a entrar al templo, sino que se queda paseando y meditando su conversación con Miles en los alrededores de éste, lo que lleva a formular la pregunta de si de verdad hay tales apariciones o si, en caso de que las haya, la joven quiere verse librada de ellas. No debe olvidarse que, ya al principio de la narración, se comenta que, cuando va a solicitar el puesto de trabajo, queda "prendada" del que va a ser su jefe y que desea, por encima de cualquier cosa, volver a verlo.

\section{LA COORDENADA ESPACIAL EN RELACIÓN A PETER QUINT Y MISS JESSEL}

Hasta el momento, la crítica especializada se ha centrado, de forma mayoritaria, en las apariciones de los "fantasmas" de Peter Quint y Miss Jessel y sus implicaciones tanto en un plano epistemológico como psicoanalítico, simbólico, etc. Tanto Quint como Miss Jessel están muertos. Su aparición en el contexto de la novela vendría a significar, por tanto, una rotura de los planos de lo real y de lo sobrenatural, y una unión de los mismos. Henry James utiliza a estos personajes para crear nuevos espacios adyacentes a la obra.

${ }^{25}$ Según el Diccionario Rioduero de Mitología griega y romana, un psicopompo se define como: Epíteto de Hermes, guía de las almas a los infiernos (Diccionario Rioduero de Mitología griega y romana, p. 210). 
Dentro de la terminología artística existe la acepción "rompimiento en gloria” para designar cuándo en una obra aparece representado el plano de lo divino, una visión del cielo ${ }^{26}$. Pero lo que hallamos en The Turn of the Screw no es, ni mucho menos, tal fenómeno, sino que vendría a ser todo lo contrario, un "rompimiento en los infiernos", pues estos dos personajes no son, precisamente, angelicales sino que, incluso en su descripción física, se presentan al lector poco menos que como demonios tentadores y corruptores de la inocencia de los niños. Numerosos teóricos, casi siempre desde el ámbito eclesial, han debatido sobre la posibilidad de la presencia de seres difuntos en el "mundo terrenal"27. Algunos, como el padre Augustin Calmet, en su Traité sur les Apparitions des Espirits, et sur les Vampires, ou les Revenans de Hongrie, Moravie, \&c. habla de esta posibilidad. En el segundo volumen de su obra, conocido como Tratado sobre los vampiros, especula también sobre la posibilidad de que los muertos vuelvan utilizando incluso su propio cuerpo, pero esa posibilidad no atañe a la circunstancia que aquí interesa ${ }^{28}$. Por otro lado, el Dr. Joseph Boneta, aun sin llegar a pronunciarse sobre la factibilidad de estas visitas, sí lo hace con respecto a la posibilidad de comunicación entre ambos mundos ${ }^{29}$. Queda, sin embargo, un espacio que podría dar satisfacción a todos estos interrogantes: el Purgatorio ${ }^{30}$. En efecto, las ánimas que poblarían ese Más Allá intermedio entre la condenación y la gloria sí pueden, según la doctrina de la Iglesia, visitar a los vivos e interceder por ellos. Por su parte, en la obra se ve cómo Quint y, especialmente, Miss Jessel, son descritos utilizando términos que podrían casar con esta posibilidad: "[...] a woman in black, pale and dreadful — with such an air also, and such a face! — on the other side of the lake" (2008:156); y, más adelante: "Dark as midnight in her black dress" (2008:196). Con respecto a la antigua institutriz se llega a insinuar que, en su "vida" actual, padece algún tipo de tormento, como ocurre en el Purgatorio ${ }^{31}$ :

${ }^{26}$ Según el Diccionario de las nobles artes: Para instruccion de los aficionados, y uso de los profesores: contiene todos los terminos... De la Pintura, Escultura, Arquitectura y Grabado... Segun el método del Diccionario de la Lengua Castellana compuesto por la Real Academia Española, editado por Diego Antonio Rejón de Silva en 1788, un rompimiento de gloria es: "Grupo de nubes, ráfagas, Ángeles o Serafines. También se dice rompimiento de país, arboleda, etc." (1788:185).

Un diccionario contemporáneo dice al respecto del término: "rotura, ruptura o rompimiento. Como en general, cualquier estado de las materias y de las formas, tiene una simbolización literal, pura traslación al mundo ideal, espiritual o psíquico del fenómeno físico correspondiente. Aquí se manifiesta la analogía paralela de los dos reinos (visible e invisible), con la mayor claridad y fuerza" (Cirlot:1998, 393).

${ }^{27}$ Como, por ejemplo, el Segundo Libro de los Macabeos (2 M 12, 46), los integrantes del Concilio de Florencia (1304), o de Trento (cf DS 1820;1580), los padres de la Iglesia Occidental San Gregorio Magno o San Juan Crisóstomo, o el referenciado Nicolás de Tolosa.

${ }^{28}$ Si sería interesante su análisis, por ejemplo, en la novela de Stephen King The Shining, donde los "fantasmas" que pueblan el Hotel Overlook sí parecen conservar su cuerpo.

${ }^{29}$ Véase su obra Gritos de el Infierno para despertar al mundo, donde las almas de los condenados, aun sin visitar nuestro mundo, sí que se ponen en contacto con los vivos para advertirles de las penas del infierno si no cambian su conducta.

${ }^{30}$ A pesar de la educación de Henry James, enmarcada en la tradición protestante, durante sus viajes por el continente europeo trabó contactos con las tradiciones religiosas de este lado del Atlántico, tanto católicas como protestantes, lo que hace que no sea descabellado que pudiera tener un conocimiento, siquiera superficial, del concepto de Purgatorio. Además, no debe olvidarse que, entre su círculo más íntimo de amigos, se encontraba el arzobispo de Canterbury, quien según el propio James le narró la anécdota en la que se basa The Turn of the Screw y quien pudo haberle puesto al corriente de los distintos estadios del Más Allá desde un punto de vista teológico.

${ }^{31}$ En el Catecismo de la Iglesia Católica se recoge un fragmento de una homilía de San Juan Crisóstomo en la que se apunta, por ejemplo, que las oraciones y las ofrendas de los vivos pueden contribuir a aliviar los tormentos 
'That she suffers the torments-!'

It was this, of a truth, that made her, as she filled out of my picture, gape. 'Do you mean', she faltered '—of the lost?'

'Of the lost. Of the damned. And that's why, to share them-' I faltered myself with the horror of it (2008:198).

De este modo, Henry James incluiría este nuevo espacio —el Purgatorio—, junto con ese "rompimiento de los infiernos", dentro de los confines de Bly, creando así un eje espacial prácticamente infinito.

Los espacios de Bly, debido quizá a la maestría narrativa de Henry James, aparecen en la literatura victoriana como ejemplos sublimes de lo que puede ser una composición matemática. Se aprecia, así, que la construcción espacial se presenta teniendo en cuenta gran parte de la tradición cultural occidental, pero, asimismo, dotándola de un alto grado de simbolismo que confiere al conjunto de la obra una perfección magnífica.

\section{CONCLUSIONES}

The Turn of the Screw se configura como un nuevo modelo en lo que a la construcción de los espacios se refiere. El propio James llegó a afirmar, en sus Notebooks [1947], que su frustrada experiencia como dramaturgo, a pesar de no reportarle los beneficios y laureles esperados, sirvió para que mejorase, entre otros aspectos, la construcción espacial de sus obras. Esto se ve magníficamente reflejado en esta novella. Henry James no parte de espacios reales, sino que concibe un espacio propio y en él focaliza el desarrollo de la trama. Bly no existe (aunque sí es un lugar verosímil) y, por ello, se presta a poder ser manipulado y construido en la forma que James lo hace: un espacio compuesto matemáticamente (composición que afecta incluso a los propios personajes) y completamente aislado del resto del mundo. Pero Henry James va más allá, procediendo a la inclusión de otro tipo de espacios que tienen más concomitancias con las interioridades psicológicas de los personajes que con la realidad geográfica. Paradójicamente, estos nuevos espacios que se crean en The Turn of the Screw se producen mediante la destrucción del plano de lo físico. La apertura hacia planos teológicos o metafísicos de la espacialidad, como se ha puesto de manifiesto, es utilizada en la prosa jamesiana para, partiendo de un espacio limitado, crear un nuevo ámbito que prácticamente carezca de naturaliza finita. Mediante la inclusión de estos nuevos espacios, Henry James abre un camino que numerosos autores del género de terror van a desarrollar a lo largo del siglo $\mathrm{xx}$, hasta llegar a nuestros días, en que dicho recurso se puede apreciar en obras tan características como, por ejemplo, las historias del ciclo de Cthulu, de H. P. Lovecraft o It (1986), de Stephen King.

a que se ven sometidas las ánimas de Purgatorio (Catecismo de la Iglesia Católica:1032, p. 241). Asimismo, la literatura también nos ofrece testimonios que apuntan en la misma dirección. La Divina Comedia de Dante sería un ejemplo paradigmático, pues en el Canto I de su "Purgatorio" se dice: "Por surcar mejor agua alza las velas/ ahora la navecilla de mi ingenio,/ que un mar tan cruel [el Infierno] detrás de sí abandona;/ y cantaré de aquel segundo reino/ donde el humano espíritu se purga/ y de subir al cielo se hace digno" (Alighieri:2007, 291, vv. 1-6). 


\section{REFERENCIAS BIBLIOGRÁFICAS}

(1992): Catecismo de la Iglesia Católica. Madrid: Asociación de Editores del Catecismo.

(1984): Diccionario Rioduero de Mitología griega y romana. Madrid: Ediciones Rioduero.

(2011): Sagrada Biblia [Versión oficial de la Conferencia Episcopal Española]. Madrid: Biblioteca de Autores Cristianos.

Alas, Leopoldo "Clarín” (2005): La Regenta. Madrid: Cátedra.

Alighieri, Dante (2007): Divina Comedia. Madrid: Cátedra.

Baquero Goyanes, Mariano (2001): Estructuras de la novela actual. Madrid: Editorial Castalia.

- (1998): ¿Qué es la novela? ¿Qué es el cuento? Murcia: Servicio de publicaciones de la Universidad de Murcia.

Boneta, Joseph (1781): Gritos de el Infierno para despertar al mundo. Madrid: Imprenta de Miguel Escribano.

Botting, Fred (2001): The Gothic. Cambridge: D. S. Brewer.

Bravo Castillo, Juan (2003): Grandes hitos de la historia de la novela euroamericana. Vol. 1 Desde sus inicios hasta el Romanticismo. Madrid: Cátedra.

- (2010): Grandes hitos de la historia de la novela euroamericana. Vol. II El siglo XIX: los grandes maestros. Madrid: Cátedra.

Brontë, Charlotte (1996): Jane Eyre. Londres: Penguin.

Calmet, Augustin (1759): Traitésur les Apparitions des Espirits, et sur les Vampires, ou les Revenans de Hongrie, Moravie, \&c.. Abadía de Senones (Francia): Chez Joseph Pariset, Imprimeur-Libraire (disponible en https://goo.gl/MMU9rQ).

- (2009): Tratado sobre los vampiros. Madrid: La Cabeza de Medusa.

Cirlot, Juan Eduardo (1998): Diccionario de símbolos. Barcelona: Ediciones Siruela.

ConRad, Joseph (1990): Heart of Darkness. Dover: Dover Publications.

Correoso Rodenas, José Manuel (2013): Dehumanized Sons at the fin-du-monde: The "Pictures" of Dorian Gray in the Context of Gothic Literature. [Trabajo final de grado defendido en julio de 2013]. Albacete, Facultad de Humanidades, Universidad de Castilla-La Mancha. Disponible en http://www.uclm.es/ab/humanidades/grado/pdf/TFG_defendidos/TFGJManuelCorreoso.pdf.

Crow, Charles L. (Ed.) (1999): American Gothic. An Anthology 1787-1916. Oxford: Blackwell.

Elis, Kate Ferguson (1989): The Contested Castle. Gothic Novels and the Subversion of Domestic Ideology. Chicago: University of Illinois Press.

Gaskell, Elizabeth (2010): The Old Nurse's Story. Whitefish (Montana): Kessinger Publishing.

Gómez Reus, Teresa y Glifford Terry (Eds.) (2013): Women in Transit through Literary Liminal Spaces. Nueva York: Palgrave McMillan.

Hart, James D. (1983): The Oxford Companion to American Literature. Oxford: Oxford University Press.

Herrero Cecilia, Juan (2000): Estética y pragmática del relato fantástico. Cuenca: Servicio de Publicaciones de la Universidad de Castilla-La Mancha.

High, Peter B. (2010): An Outline of American Literature. Nueva York: Longman.

Hogle, Jerrold E. (Ed.) (2011): The Cambridge Companion to Gothic Fiction. Cambridge: Cambridge University Press.

JAMES, HenRy (2011): Daisy Miller, Otra vuelta de tuerca y otros relatos. Madrid: Espasa-Calpe.

- (1987): The Complete Notebooks of Henry James. Oxford: Oxford University Press.

- (2011): The Portrait of a Lady. Londres: Penguin.

- (2008): The Turn of the Screw and Other Stories. Oxford: Oxford University Press.

KING, STEPHEN (1987): It. Nueva York: Signet.

- (2012): The Shining. Londres: Hodder \& Stoughton. 
Las Heras, Antonio: "Importancia de los rituales iniciáticos en la Masonería”. Disponible en: https://drantoniolasheras.wordpress.com/2015/09/22/importancia-de-los-rituales-iniciaticos-en-la-masoneria-por-el-prof-dr-antonio-las-heras/

Le Goff, Jaques (1989): El nacimiento del Purgatorio. Madrid: Taurus.

Lloyd-Smith, Allan: "Nineteenth-Century American Gothic", In Punter, David (Ed.) (2000): A Companion to the Gothic. Oxford: Blackwell: pp. 109-121.

López SAntos, Miriam (2010): "Ampliación de los horizontes cronotópicos de la novela gótica”, en Signa, n. ${ }^{\circ} 19$, pp. 273-292.

Lusting, T. J.: "Introduction", en James, Henry (2008): The Turn of the Screw and Other Stories. Oxford: Oxford University Press, pp. vi-lv.

Manrique, Jorge (2003): Coplas a la muerte de su padre. Madrid: Castalia.

Punter, David (Ed.) (2000): A Companion to the Gothic. Oxford: Blackwell.

RAdCLIfFe, AnN (1998): The Mysteries of Udolpho. Oxford: Oxford University Press.

Rejón de Silva, Diego Antonio (1788): Diccionario de las nobles artes: Para instrucción de los aficionados, y uso de los profesores: contiene todos los términos... De la Pintura, Escultura, Arquitectura y Grabado... Según el método del Diccionario de la Lengua Castellana compuesto por la Real Academia Española. Madrid: Imprenta de D. Antonio Espinosa.

Rodríguez Guerrero-Strachan, SAntiago: "Introducción” a James, Henry (2011): Daisy Miller, Otra vuelta de tuerca y otros relatos. Madrid: Espasa-Calpe.

Ruland, Richard y Bradbury, Malcolm (1992): From Puritanism to Postmodernism. A History of American Literature. Nueva York: Penguin.

Tolosa, Nicolás DE (1918): El Purgatorio. Barcelona: Bloud y Gay Editores.

Wharton, Edith (1997): The Ghost Stories of Edith Wharton. Nueva York: Scribner.

Wheatley, Helen (2006): Gothic Television. Manchester: Manchester University Press. 
